\title{
Estudio de las propiedades ópticas de recubrimientos Sol-Gel dopados con fluoresceína en función de la concentración y del pH
}

\author{
E.F. MONTERO', M. A. GARCÍA'1, M. A. VILLEGAS², J. LLOPIS'1 \\ ${ }^{1}$ Depto. Física de Materiales, Fac. de Ciencias Físicas, Universidad Complutense de Madrid. Madrid, España \\ ${ }^{2}$ Centro Nacional de Investigaciones Metalúrgicas, CSIC. Madrid, España
}

\begin{abstract}
Se investiga la variación con el pH de las propiedades ópticas (absorción y luminiscencia) de moléculas de fluoresceína incorporadas a recubrimientos porosos sol-gel en un intervalo de concentraciones comprendido entre 0,5 y 3,8 \% en peso. Los espectros de absorción de los recubrimientos presentan bandas principales en $435 \mathrm{~nm}$ y $487 \mathrm{~nm}$ para $\mathrm{pH}$ ácido y básico, respectivamente. Al comparar con los espectros de las disoluciones, la posición de ambos máximos se desplaza hacia el rojo y aparecen en $438 \mathrm{~nm}$ y $492 \mathrm{~nm}$. La dependencia de la absorción de la fluoresceína en recubrimientos y disoluciones se ajusta a la ley de Lambert-Beer. Al excitar los recubrimientos y las disoluciones con fotones de la región en que la absorción es importante, los espectros de fotoluminiscencia muestran una banda ancha principal en $520 \mathrm{~nm}$, tanto para $\mathrm{pH}$ ácido como para básico. En este caso y contrariamente a lo observado en los espectros en disolución, la intensidad a $520 \mathrm{~nm}$ decrece con el pH al aumentar éste desde 2 a 12.
\end{abstract}

Palabras clave: fluoresceína, $p H$, recubrimientos sol-gel, absorción, fotoluminiscencia

\section{Study of optical properties of fluorescein-doped sol-gel coatings as a function of concentration and $\mathrm{pH}$}

Optical properties (absorption and luminescence) of fluorescein molecules encapsulated in porous sol-gel coatings for concentrations ranging from 0.5 to $3.8 \mathrm{wt} \%$ are investigated as a function of $\mathrm{pH}$. Absorption spectra of the coatings show main bands at $435 \mathrm{~nm}$ and 487 $\mathrm{nm}$ for acid and basic $\mathrm{pH}$, respectively. Both peaks appear in the solutions of fluorescein redshifted at $438 \mathrm{~nm}$ and $492 \mathrm{~nm}$. However, the dependence in intensity of both peaks as a function of $\mathrm{pH}$ is found essentially similar between coatings and solutions. In both cases, the absorption dependence on concentration can be fitted to the Lambert-Beer law. On the other way, photoluminescent spectra of coatings and solutions upon excitation in the peak absorption region, show a dominant broad band peaked at $520 \mathrm{~nm}$ for both acid and basic $\mathrm{pH}$. The intensity of the $520 \mathrm{~nm}$-coating band displays a decreasing behaviour with increasing $\mathrm{pH}$ from 2 to 12 , in contrast with those found for the same peak when the corresponding solutions were analysed.

Key Words: fluorescein, $\mathrm{pH}$, sol-gel films, absorption, photoluminescence

\section{INTRODUCCIÓN}

La fluoresceína es una molécula orgánica que en disolución líquida presenta importantes propiedades ópticas tanto de absorción como de luminiscencia, dando espectros característicos en la zona del visible, que se ven modificados con el $\mathrm{pH}[1,2,3]$. Las propiedades de la fluoresceína han permitido que se utilice como marcador óptico en biología [4] y que actualmente se pretenda explotar como indicador óptico (absorción y luminiscencia) de $\mathrm{pH}[2,3,5]$. En el desarrollo de sensores ópticos, la incorporación de moléculas orgánicas a una matriz porosa de tipo sol-gel [6-8] resulta ventajosa, ya que se evitan o disminuyen algunos de los inconvenientes de su uso en disolución, tales como procesos de contaminación y degradación de la molécula, procesos no radiativos, etc., además de ofrecer la posibilidad de ser reutilizados. Otras ventajas que presentan los recubrimientos sol-gel son la relativa facilidad de preparación, el bajo coste, la gran resistencia química, la buena adherencia mecánica, y la aptitud del sol para recubrir muchos tipos de superficies. Todo ello ha permitido el desarrollo de esta técnica en nuevos sistemas que presentan posibilidades prometedoras en aplicaciones de interés tecnológico $[9,10]$.

En este trabajo se estudian y comparan las propiedades ópticas de la fluoresceína en recubrimientos sol-gel de sílice y en disoluciones, haciendo especial énfasis en estudiar las propiedades ópticas al variar la concentración de fluoresceína y el $\mathrm{pH}$ del medio en que se encuentra.

\section{MÉTODO EXPERIMENTAL}

En la preparación de los recubrimientos se utilizaron como precursores tetraetóxido de silicio (TEOS), ácido clorhídrico 3M y metanol en proporciones molares 1:4:8, respectivamente. La fluoresceína se añadió al sol disuelta en metanol para obtener recubrimientos con concentraciones de 0,51, 0,97, 1,88, 2,82 y 3,77 \% en peso. En el cálculo de las concentraciones molares se utilizó una densidad media para los recubrimientos de $1,5 \mathrm{~g} / \mathrm{cm}^{3}$. Como sustratos se utilizaron portas comerciales de vidrio sódico cálcico con espesores de $0,5 \mathrm{~mm}$, que previamente se limpiaron cuidadosamente con etanol.

Los recubrimientos sol-gel sobre los portas, se prepararon por la técnica de inmersión-extracción, obteniéndose diferentes espesores al extraerlos a distintas velocidades (entre 5 y $25 \mathrm{~cm} / \mathrm{min}$ ). Los recubrimientos así obtenidos se dejaron secar al aire al menos una hora antes de introducirlos en una estufa donde permanecieron tres días a $60^{\circ} \mathrm{C}$. Posteriormente, se determinaron los espesores de los recubrimientos a partir de los espectros de reflexión [11].

Las disoluciones con fluoresceína se prepararon empleando metanol y disoluciones tampón comerciales de $\mathrm{pH}$ entre 2 y 12 (pHydrion Buffers, Micro Essential Laboratory) en proporciones de volumen 1:4, respectivamente. Las disoluciones de $\mathrm{pH}<2$ se prepararon con $\mathrm{HCl}$ y las de $\mathrm{pH}>12$ con $\mathrm{NaOH}$. Para obtener los espectros de las disoluciones, se utilizó una cubeta de cuarzo de $1 \mathrm{~cm}$ de espesor.

Los recubrimientos se hidrataron durante $1 \mathrm{~h}$ en agua destilada 
para facilitar los posteriores procesos de protonación y desprotonación. Seguidamente, las muestras se mantuvieron durante 5 min sumergidas en disoluciones tampón de $\mathrm{pH}$ crecientes entre 0 y 13,5. A continuación se obtuvieron los espectros de absorción óptica y de luminiscencia de las muestras tal y como se ha descrito en trabajos anteriores $[7,12]$.

\section{RESULTADOS EXPERIMENTALES Y DISCUSIÓN}

Los espectros de absorción de los recubrimientos presentan dos bandas anchas con máximos principales en 435 y $487 \mathrm{~nm}$, como se muestra en la Fig. 1a. La intensidad relativa de ambas bandas depende del $\mathrm{pH}$ de la disolución en la que previamente se sumergieron los recubrimientos. Así, para medios con $\mathrm{pH}$ ácidos la contribución al espectro de la banda de $435 \mathrm{~nm}$ es mayor que la de $487 \mathrm{~nm}$, ocurriendo lo contrario para $\mathrm{pH}$ básicos. La Fig. $1 \mathrm{~b}$ muestra los espectros de la fluoresceína en disolución metanol-tampón que, aunque similares en su forma, presentan variaciones en la intensidad relativa de los máximos y en la longitud de onda en la que aparecen, especialmente a $\mathrm{pH} 0,5$ y 13,5 . Así, a pH 0,5 el máximo de absorción en las disoluciones aparece a 438 $\mathrm{nm}$ en vez de a $435 \mathrm{~nm}$. Por otro lado, a pH 13,5 la disolución muestra el máximo a $492 \mathrm{~nm}$ en vez de a $487 \mathrm{~nm}$ (Figs. 1 a y b). Este desplazamiento en la posición del máximo hacia el rojo, respecto del observado en el recubrimiento, es similar al encontrado por Shamansky [2], y posiblemente relacionado con un estado electrónico molecular más confinado, que se vería favorecido por la transición del estado sol al estado vítreo del recubrimiento, mucho más rígido. La forma lactónica de la fluoresceína en disolución presenta una mínima absorción [1,2] cerca de $\mathrm{pH} 3$, que no resulta tan marcada en el recubrimiento, lo que sugiere que la red de sílice afecta la estructura y los niveles electrónicos de la fluoresceína encapsulada en los poros abiertos.

La Fig.2 muestra la variación con el pH de la intensidad de absorción para los dos máximos principales de la fluoresceína en el recubrimiento, donde se puede apreciar que cada máximo sigue un comportamiento distinto. La intensidad de la banda de $487 \mathrm{~nm}$ aumenta con el pH y su variación relativa es mayor que la observada para la banda de $435 \mathrm{~nm}$, la cual disminuye en intensidad al aumentar el pH. Para la banda de $487 \mathrm{~nm}$, se observa un comportamiento aproximadamente lineal de la absorción con el $\mathrm{pH}$ en el intervalo entre 4 y 10. Por otro lado, la variación de la banda de $435 \mathrm{~nm}$ se puede aproximar a dos segmentos lineales, comprendidos entre $\mathrm{pH} 0,1$ y 7,0 y otro para $\mathrm{pH}$ entre 7,0 y 13,5 . Este comportamiento difiere del que se obtiene en disolución, donde el máximo de $438 \mathrm{~nm}$ muestra una disminución en intensidad con el $\mathrm{pH}$ seguida de una recuperación parcial para $3 \leq$ $\mathrm{pH} \leq 5$, volviendo a disminuir para $\mathrm{pH}$ mayores. Los parámetros de los ajustes lineales se resumen en la Tabla I. Estos resultados permiten considerar a este dispositivo como un buen candidato para desarrollar un optodo sencillo de $\mathrm{pH}$, similar al que se ha desarrollado con otras sustancias dopantes $[6,7,14]$.

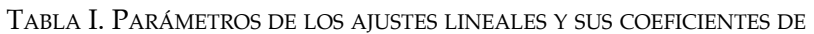
CORRELACIÓN QUE APARECEN EN LAS FIGURAS QUE SE INDICAN.

\begin{tabular}{|c|c|c|c|c|c|}
\hline \multirow{2}{*}{ Figura } & \multirow{2}{*}{$\mathrm{pH}$} & \multicolumn{2}{|c|}{$\mathrm{y}=\mathrm{mx}+\mathrm{b}$} & \multirow{2}{*}{$\mathrm{r}^{2}$} & \multirow{2}{*}{ Comentario } \\
\cline { 3 - 5 } & & $\mathrm{m}$ & $\mathrm{b}$ & & \\
\hline 2 & $0,1-7,0$ & $-5,246 \times 10^{-3}$ & 0,10022 & 0,9634 & Banda de $435 \mathrm{~nm}$ \\
\hline 2 & $7,0-13,5$ & $-1,449 \times 10^{-3}$ & 0,07535 & 0,9483 & Banda de $435 \mathrm{~nm}$ \\
\hline 2 & $4,0-10,0$ & $9,450 \times 10^{-3}$ & 0,01614 & 0,9954 & Banda de 487 nm \\
\hline 3 & $1,5-10,0$ & $-8,299 \times 10^{-2}$ & 1,1249 & 0,9960 & Emis. recub.(520)-pH \\
\hline $4 \mathrm{a}$ & 6,2 & 0,39065 & 0,04389 & 0,9970 & Ley Beer disolución \\
\hline $4 \mathrm{~b}$ & 7,0 & $1,039 \times 10^{-3}$ & 0,01139 & 0,9843 & Ley Beer recubrim. \\
\hline $5 \mathrm{a}$ & --- & --- & --- & 0,7377 & Emisión recub.(520) \\
\hline $5 \mathrm{~b}$ & --- & --- & --- & 0,9937 & Emisión disol.(520) \\
\hline
\end{tabular}

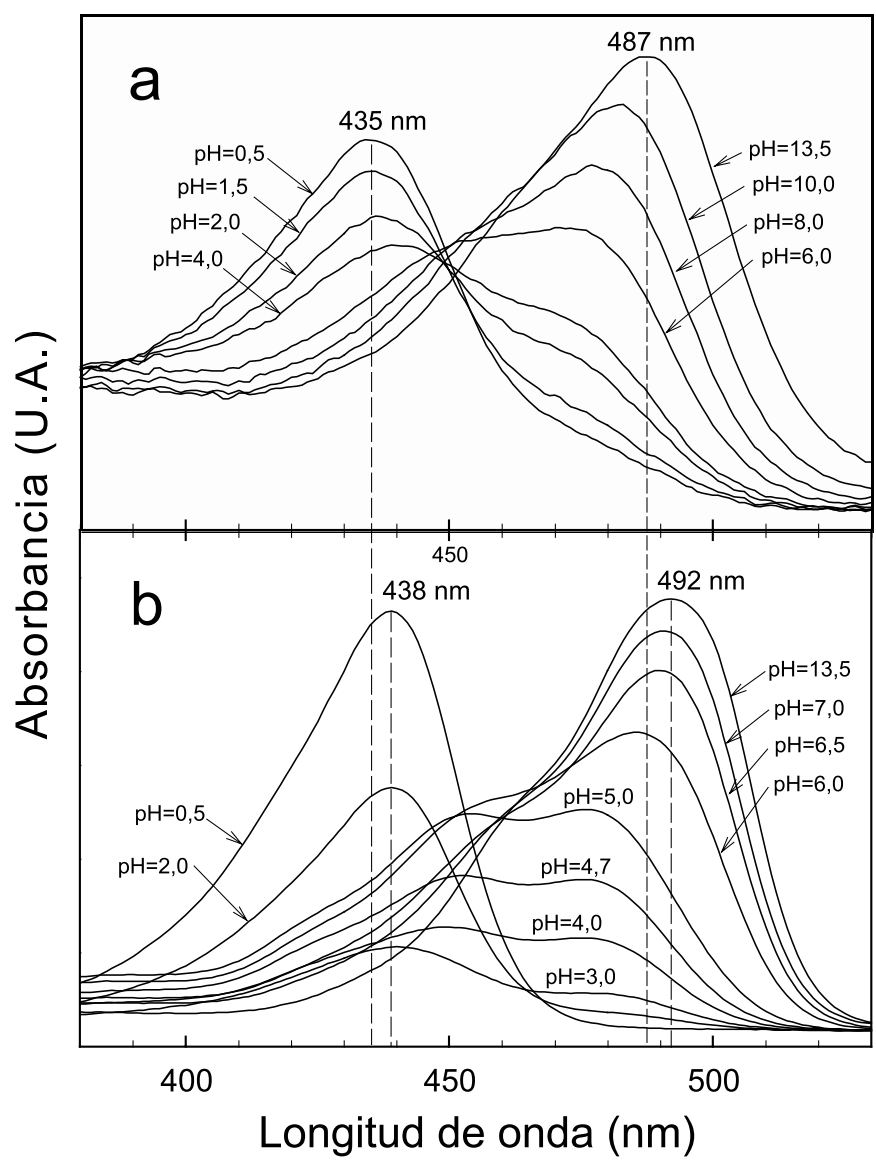

Fig 1. Espectros de absorción de la fluoresceína para distintos $\mathrm{pH}$ en (a) el recubrimiento con $0,97 \%$ en peso y $245 \mathrm{~nm}$ de espesor; (b) disoluciones.

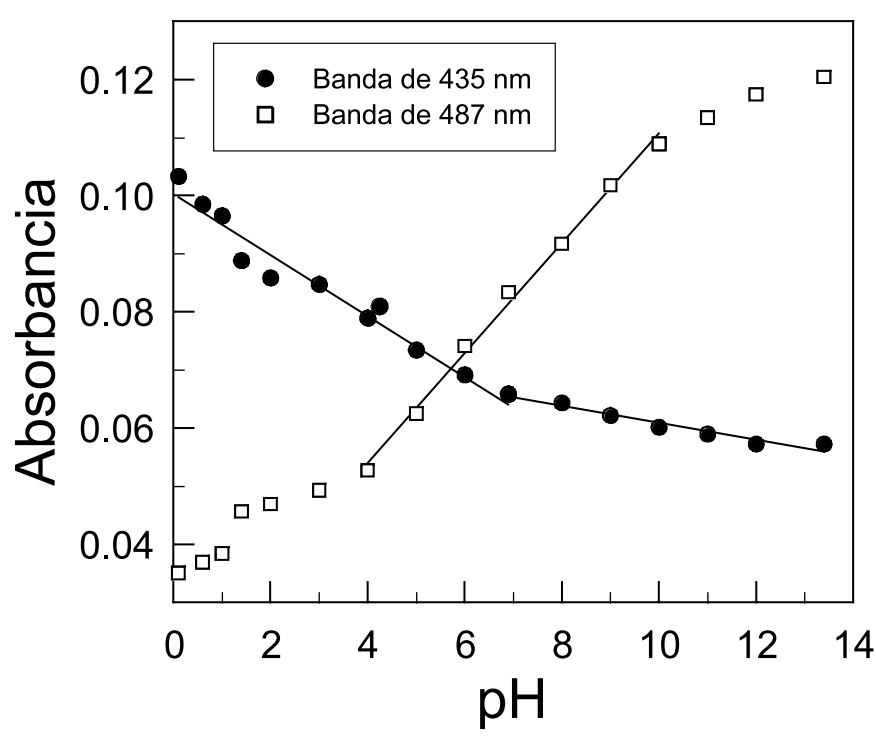

Fig 2. Evolución de la absorbancia con el $\mathrm{pH}$ de las bandas de 435 nm (ácida) y 487 nm (básica) de la fluoresceína ( $0,97 \%$ en peso) en el recubrimiento de $245 \mathrm{~nm}$ de espesor. 


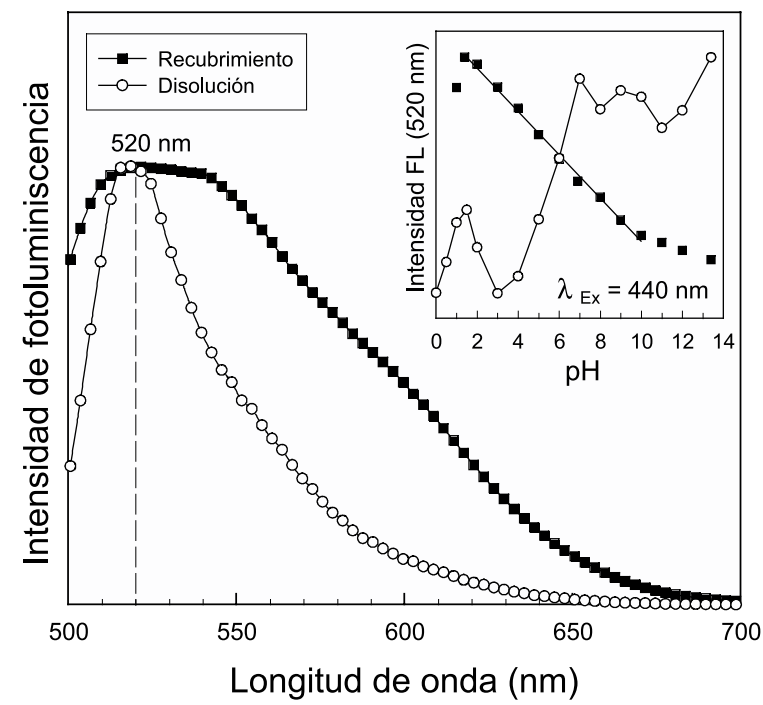

Fig 3. Espectros normalizados de luminiscencia de la fluoresceína en disolución y en recubrimiento para una excitación de $440 \mathrm{~nm}$ a pH 13,5. La figura insertada muestra la evolución normalizada de la emisión de $520 \mathrm{~nm}$ con el $\mathrm{pH}$ para disoluciones y recubrimientos (ajuste lineal entre $\mathrm{pH} 1,5$ y 10,0$)$.

Una característica del espectro de emisión de la fluoresceína en los recubrimientos a un $\mathrm{pH}$ de 13,5 y al excitar con fotones de $440 \mathrm{~nm}$, es que presentan un perfil claramente más ancho que en disolución (Fig. 3). Esta diferencia se relaciona con el carácter compuesto del espectro de emisión de los recubrimientos que presenta dos bandas de emisión secundarias alrededor de 550 y $600 \mathrm{~nm}$ (Fig. 3). Estas emisiones de menor energía son probablemente la contribución de una fracción de mo-
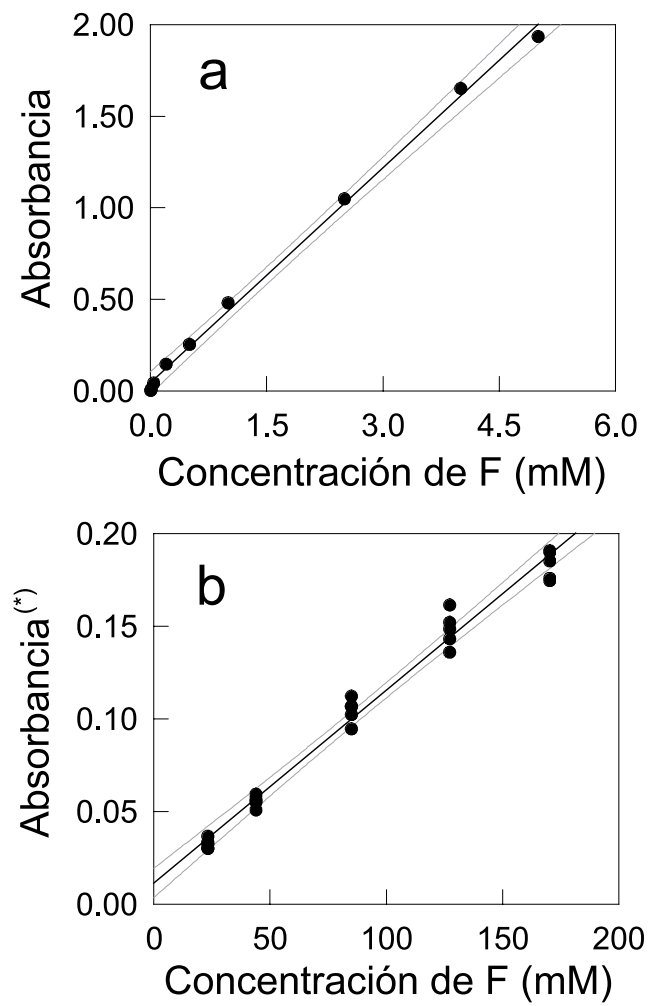

Fig 4. Evolución del máximo de absorbancia con la concentración de fluoresceína en (a) disoluciones de etanol $(486 \mathrm{~nm}$ ) y (b) recubrimientos a $\mathrm{pH} 7,0(477 \mathrm{~nm})$. Se muestran las rectas de mejor ajuste y los intervalos de confianza del $99 \%$. $\left(^{*}\right)$ Absorbancia / Espesor. léculas de fluoresceína que permanecen atrapadas en poros cerrados y que conservan el estado más protonado, correspondiente a la catálisis ácida del sol. Esta interpretación se basa en que en un medio ácido los espectros de luminiscencia de la fluoresceína presentan unas componentes secundarias similares a las mencionadas en 550 y $600 \mathrm{~nm}$.

En el gráfico insertado en la Fig. 3, se muestra el comportamiento lineal monótono con el pH de la emisión de fluoresceína en el recubrimiento, lo que sugiere su aplicación como optodo luminiscente de $\mathrm{pH}$. Además, en el recubrimiento el intervalo de $\mathrm{pH}$ en el que presenta la respuesta lineal (de 1,5 a 10) es mucho mayor que en disolución (de 4 a 7) lo que representa una mejora respecto al comportamiento de la disolución. Los parámetros del ajuste se recogen en la Tabla I.

Tanto en los recubrimientos como en las disoluciones (Figs. 4a y 4b), la intensidad del máximo de absorción aumenta proporcionalmente con la concentración de fluoresceína, lo que indica la validez de la ley de Lambert-Beer para este intervalo de concentraciones (Tabla I). No obstante, dado que la fluoresceína emite luz con una gran eficiencia cuántica a la vez que la absorbe, la medida de la intensidad del máximo de absorción se vería influida por la emisión luminiscente. Esto significaría que los valores medidos directamente del espectro de absorción serían en realidad algo menores y necesitarían una corrección. Sin embargo y afortunadamente, este efecto se comprueba que es pequeño y en cualquier caso la tendencia lineal de la luminiscencia con la concentración (Fig. 5a) influiría dando también una respuesta lineal. En la Fig. 5b se muestra la variación lineal de la intensidad de luminiscencia de las disoluciones con la concentración, aunque en este caso con una pendiente positiva. La variación de la absorción (emisión) dividida por el espesor utilizada en la Fig. 4b (5a) confirma que tanto la absortividad molar (eficiencia cuántica) como la concentración se ven poco influidas por el espesor del recubrimiento. En la Tabla I se indican los valores de los ajustes mostrados en las Figs. 5a y $5 \mathrm{~b}$.
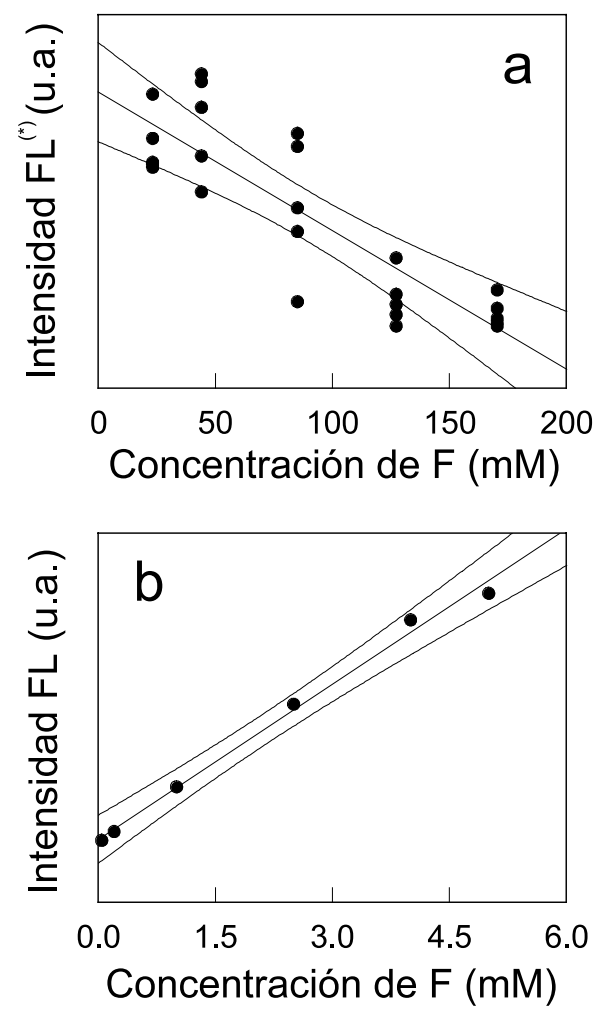

Fig 5. Intensidad de la luminiscencia a 520 nm en función de la concentración de fluoresceína al excitar con $440 \mathrm{~nm}$ en (a) recubrimientos a pH 7,0 y (b) disoluciones de etanol. Se muestran las rectas de mejor ajuste y los intervalos de confianza del $99 \%$. $\left(^{*}\right)$ Luminiscencia / Espesor. 


\section{CONCLUSIONES}

Los resultados señalan que la incorporación de la fluoresceína en recubrimientos vítreos de tipo sol-gel, ofrece ventajas y buenas posibilidades de aplicación en sensores ópticos de $\mathrm{pH}$ (luminiscentes y de absorción) para un amplio intervalo de $\mathrm{pH}$.

En los recubrimientos dopados con fluoresceína hay mayor linealidad en la variación de la intensidad de la absorción y la luminiscencia que en las disoluciones.

Al incorporar la fluoresceína a los recubrimientos sol-gel, ésta se mantiene sensible al $\mathrm{pH}$ del entorno y esto permite utilizar esos recubrimientos como optodos de $\mathrm{pH}$.

Para las concentraciones estudiadas, la absorción de los recubrimientos sigue una ley lineal de tipo Lambert-Beer.

\section{AGRADECIMIENTOS}

Los autores desean dar su agradecimiento al proyecto de la DGI MAT2002-04503-C02-02. Uno de los autores desea agradecer especialmente la beca del CONICIT de Costa Rica para sufragar parcialmente sus estudios doctorales en España.

\section{BIBLIOGRAFÍA}

1. M.R.S. Fuh, L.W. Burgess, T. Hirschfeld, G.D. Christian, F. Wang. "Single fibre optic fluorescence $\mathrm{pH}$ probe". Analyst. 112, 1159-1163 (1987).

2. L.M. Shamansky, M. Yang, M. Olteanu, E.L. Chronister. “A spectroscopic study of the $\mathrm{pH}$ sensor response of fluorescein doped silica and aluminosilica sol-gel glasses". Mat. Lett., 26, 113-120 (1996).

3. M.D. Gulcev, G.L.G. Goring, M. Rakic, J.D. Brennan. "Reagentless pHbased biosensing using a fluorescently-labelled dextran co-entrapped with hydrolytic enzyme in sol-gel derived nanocomposite films". Anal. Chim. Acta, 457, 47-59 (2002).

4. R.D. Lillie. “Conn's biological stains" 9th Ed., Williams \& Wilkins, Baltimore, USA (1977).

5. B.D. MacCraith, V. Ruddy, C. Poter, B. O'Kelly, J.F. McGilp. “Optical waveguide sensor using evanescent wave excitation of fluorescent dye in sol-gel glass". Electron. Lett. 27 [14] 1247-1248 (1991).

6. J. Lin, O. Liu. "An optical $\mathrm{pH}$ sensor with a linear response over a broad range". Anal. Chim. Acta, 408, 49-55 (2000).

7. M.A. García, S.E. Paje, M.A. Villegas, J. Llopis. "Preparation and characterization of calcein-doped thin coatings". Appl. Phys. A, 74, 83-88 (2002).

8. E. Bescher, J.D. Mackenzie. "Hybrid organic-inorganic sensors". Mat. Sci. Eng. C, 6, 145-154 (1998).

9. C.J. Brinker, G.W. Scherer. "Sol-Gel Science. The Physics of Sol-Gel Processing". Academic Press, Capítulo 14, pp 839-873 (1990).

10. D.R. Uhlmann, G.Teowee. "Sol-gel science and technology: current state and future prospects". J. Sol-Gel Sci. Tech., 13, 153-162 (1998).

11. M.A. García, S.E. Paje, J. Llopis, M.A. Villegas. “Influencia de las condiciones de preparación en la luminiscencia de recubrimientos de sílice pura". Bol. Soc. Esp. Ceram. y V., 39, [5] 641-646 ( 2000).

12. M.A. Villegas, L. Pascual, S.E. Paje, M.A. García, J. Llopis. "Eriochrome cyanine doped sol-gel coatings. Optical behavior against $\mathrm{pH}^{\prime \prime}$. J. Europ. Ceram. Soc. 20, 1621-1628 (2000).

13. A. Lobnik, I. Oehme, I. Murkovic, O.S. Wolfbeis."pH optical sensors based on sol-gels: Chemical doping versus covalent immobilization". Anal. Chim. Acta, 367, 159-165 (1998).

14.D.A. Nivens, Y. Zhang, S.M. Angel. "A fiber-optic pH sensor prepared using a base-catalized organo-silica sol-gel". Anal. Chim. Acta, 376, 235-245 (1998).

Recibido: 01.02 .03

Aceptado: 30.11 .03 